Nicole Marchand-Zañartu et Jean Lauxerois, 32 grammes de pensée. Essai sur l'imagination graphique, Paris, MÉDIAPOP Éditions, 2020, 155 p.

\title{
Aurèle Crasson
}

\section{(2) OpenEdition} Journals

Édition électronique

URL : https://journals.openedition.org/genesis/5919

DOI : 10.4000/genesis.5919

ISSN : 2268-1590

Éditeur :

Presses universitaires de Paris Sorbonne (PUPS), Société internationale de génétique artistique littéraire et scientifique (SIGALES)

\section{Édition imprimée}

Date de publication : 1 juillet 2021

Pagination : 213-214

ISBN : 979-10-231-0710-4

ISSN : $1167-5101$

\section{Référence électronique}

Aurèle Crasson, « Nicole Marchand-Zañartu et Jean Lauxerois, 32 grammes de pensée. Essai sur l'imagination graphique, Paris, MÉDIAPOP Éditions, 2020, 155 p. », Genesis [En ligne], 52 | 2021, mis en ligne le 01 juillet 2021, consulté le 10 octobre 2022. URL : http://journals.openedition.org/genesis/ 5919 ; DOI : https://doi.org/10.4000/genesis.5919 


\section{Comptes rendus d'ouvrages}

Nicole Marchand-Zañartu et Jean Lauxerois, 32 grammes de pensée. Essai sur l'imagination graphique, Paris, MÉDIAPOP Éditions, 2020, 155 p.

\section{Compte rendu par Aurèle Crasson*}

Nicole Marchand-Zañartu et Jean Lauxerois reviennent, neuf ans après leur première publication 1 , sur les saisissantes représentations des «jaillissements » de pensée. Dans ce nouvel opus, ils donnent à voir la naissance, la construction et l'invention sur le papier d'une pensée non encore conceptualisée, non encore codée, celle de trente-deux créateurs, poètes, philosophes, mathématiciens ou encore musiciens. Ces images de traits, de lignes et surfaces colorées, de schémas, de dessins dans lesquels parfois se glissent, comme un avant «avant-dire», quelques lettres ou quelques indications pour un projet d'écriture, s'accompagnent de trente-deux textes des auteurs qui guident le lecteur, lui offrant «un libre fil d'Ariane dans ces fascinants labyrinthes ».

Le titre surprend à plusieurs égards. On pourrait croire qu'il s'agit des 32 grammes qui correspondent aux 11 grammes de plus que les 21 supposés du poids de l'âme principe vital et spirituel qui anime le corps humain, or les auteurs de 32 grammes de pensée choisissent le «gramme» dans son sens étymologique, «signe» ou «écrit», pour désigner les représentations schématiques de pensées fulgurantes évitant à celles-ci de rejoindre les $90 \%$ de rêves oubliés au sursaut du matin.

En s'arrêtant encore davantage sur ce vocable pris dans l'acception qui le détermine dans ce volume, notons que le mot «grammage» est aussi une référence au poids du support d'inscription, lui bien tangible. Ce terme «grammage » désigne la masse surfacique du papier, sa force dit-on encore, ce qui permet à l'image de pensée d'advenir. Le trait n'est donc jamais séparé du poids de sa représentation que porte le papier. Nicole Marchand-Zañartu et Jean Lauxerois font d'ailleurs référence au gramme comme unité de mesure. «Mesure de la légèreté » dit ainsi la préface du livre un millième de kilogramme, soit très peu en effet par rapport à la sidération de l'image produite.

Mais, ramené au volume des éditions MÉDIAPOP, 32 grammes de pensée ne pèse pas les $0,032 \mathrm{~kg}$ de l'unité universelle. Et pour cause, les auteurs y ont ajouté, pour chaque dessin d'auteur, une interview, une référence historique, une analyse matérielle; ils guident le lecteur sans gêner sa propre interprétation, le mènent à s'interroger. Ils livrent là, via de multiples voix, un livre «pesamment» pensé!

«Traces-tracés », une relation dans laquelle réside une bonne part des enjeux de la critique génétique. Les auteurs ne font cependant pas ici œuvre de génétique, ils donnent à voir un «gramme» de trace de création, le gramme natif, initial, auquel remontent tous les autres.

Le livre lui-même surprend par sa mise en page, à commencer par l'index des grammes esthétiquement composé. Surprenant aussi est le choix des catégories. La tentation d'un classement ne prive-t-elle pas déjà ces gestes de leur singularité? Alors quel est l'enjeu de ce livre? Comment se donne-t-il à consulter? Qu' insuffle-t-il ?

Les intérêts sont multiples. 32 grammes s'adresse à l'esthète. Voir le gramme 32 de Claude Simon si «plastique» qu'il en couvre le volume, le gramme $9 \mathrm{du}$ coloriste Julius O. Bluemner, le «Vermillonaire», dont on remarquera le texte "étouffé » sous le poids d'une large palette des couleurs, ou encore le minimaliste dessin de Charcot, gramme 10, qui rappelle le «sexualschema» dressé par Freud en 1895 dans le manuscrit $\mathrm{G}$, et qui figurait dans le précédent volume des Images de pensées, publié par Nicole Marchand-Zañartu et Marie-Haude Caraës en 2011.

32 grammes s'adresse aussi au musicien. Ainsi le gramme 26 de Luigi Nono surprend (encore) par la transposition poétique décalée de sa notation. Il ne s'agit pas d'un jeu; quiconque a vu les manuscrits de Nono comprendra que les choix de courbes et de couleurs de ses papiers participent pleinement de l'engagement de son œuvre. György Ligeti (gramme 23) se montre en topo-géographe de la musique. Si le pays imaginaire, «Kilviria», que le musicien a dessiné à l'âge de cinq ans, constitue une représentation trop élaborée pour être une «fulguration graphique», cette image rassemble l'univers musical imprégné des requiem, berceuses et chants populaires de son enfance. Image de «rêve originel » qui, même créée dans un temps plus long de graphie, demeure une composition «matrice» où tout créateur puise aujourd'hui encore son inspiration, comme ce fut le cas pour Ted Nelson, ni musicien ni poète mais inventeur de l'hypertexte.

Quel lecteur de Valéry peut rester insensible à cette représentation si abstraite (gramme 30), provenant du fonds Paul Valéry de la Bibliothèque nationale de France? Bien qu'influencée par la mise en page d'Un coup de dé jamais n'abolira le hasard de Mallarmé, elle n'en exprime pas moins une volonté de s'exiler du geste rituel de l'écriture, un «laisser-filer» du trait mû

\section{(*) ITEM.}

1. Nicole Marchand-Zañartu et Marie-Haude Caraës, Images de pensée, Réunion des Musées Nationaux, janvier 2011. Les deux auteures de ce volume avaient publié une chronique intitulée «Penser avec» dans le numéro 37 de Genesis, «verbal - non verbal», 2013 (en ligne sur openedition.org). 
par une pensée libre d'associations, hors de toutes convenances.

Sont concernés aussi bien les architectes que les musiciens, les peintres, les écrivains, les botanistes et même les cuisiniers, tel Ferran Adrià, un des meilleurs chefs au monde. Mais passé cet intérêt particulier, ce volume s'adresse au lecteur universel à qui il montre que la pensée, avant d'être langage verbal, est peut-être comme le disent Nicole Marchand-Zañartu et Jean Lauxerois « dans son frémissement initial, [...] d'emblée image» mais pas seulement.

Ce «frémissement initial» fait appel non pas à ces représentations usuelles : dessin, trait, ou image, mais justement à une projection exempte de codification, une idiosyncrasie qui, pour le peintre, le botaniste, l'architecte, met l'éclair de la pensée pure à distance d'une pensée captive des modes conventionnels de la représentation.

Mais que faire de telles images dans la démarche d'un généticien du texte? L'analyse génétique tente d'observer l'origine, le sillage tracés par une œuvre, son contexte, sa limite.

Si les images de pensées ne sont pas des œuvres, elles participent à leur devenir. «Figure, schème, schéma, dessin, dessin préparatoire, esquisse, croquis...» pour reprendre quelques-uns des vocables utilisés dans la postface de Nicole MarchandZañartu et Marie-Haude Caraës ${ }^{2}$, elles sont tout cela à la fois et tout autre chose. Il faut donc s'attacher à les observer avec un regard neuf, juste observer ce qu'elles diffusent en nous de compréhension de la création car on ne peut pas en rester à cette supposée origine. Il faut la confronter au contexte de sa production.

Deux questions essentielles auxquelles N. Marchand-Zañartu et Jean Lauxerois tentent d'apporter leur éclairage et conduisent le lecteur arrivé au terme de sa consultation et au seuil de la postface de 32 grammes : 1) Ces matériaux sont-ils des images? 2) Qu'est-ce qui nous permet de signifier que ces images seraient «de pensée»?

Jean Lauxerois, spécialiste de la pensée grecque et dont le travail porte entre autres sur le sens de l'œuvre d'art et de la culture, livre dans cette postface une réflexion philosophique sur la production de ces images, convoquant dans sa démonstration «le beau sans schème » de Socrate, «l'imagination au cœur du fonctionnement de la raison » chez Kant, aussi bien que la pensée de Benjamin pour qui «le gramme concrétise l'image de pensée comme telle, laquelle peut même apparaître sous trois modes grammatiques différents, en prenant ainsi plusieurs sens».

32 grammes est plus qu'un livre d'images, c'est une question en abyme, celle de la création avant la création, de l'origine avant une origine supposée, et d'autant plus un livre pour les généticiens que «le gramme n'est pas une œuvre : il est (son) l'amorce, (sa) une greffe initiale». Leçon à méditer !

Alain Boillat, En cas de malheur de Simenon à Autant-Lara. Essai de génétique scénaristique, Genève, Droz, 2020, 376 p.

\section{Compte rendu par Olivier Lumbroso*}

Dans le domaine des études sur l'adaptation cinématographique des œuvres littéraires, l'essai d'Alain Boillat propose une démarche novatrice sur plusieurs plans théoriques et analytiques. Il se fonde, en effet, sur une approche génétique globale des scénarios, manuscrits et tapuscrits d'En cas de malheur (1956) de Georges Simenon. Cet ensemble se voit saisi dans l'enchaînement entrelacé des diverses campagnes d'écriture qui produisent ces écrits préparatoires : "premier incipit», «plans scénariques », «scénarios partiels», «continuités dialoguées» et «scénario de tournage ». Ainsi, sont décrites et interprétées les traces de la genèse scénaristique souvent négligées par les études cinématographiques : la matérialité des supports et des graphies (papier, recto/verso, écritures linéaire/tabulaire, didascalies...), la datation et la temporalité relative (séquentialité, segmentation, montage et rythme, scènes et plans, flash-backs...), les fonctions de régie et de mise en scène (diégèse, décor, éclairage, habillement, ton...), l'évaluation des coûts (production, bobines, studios...), la complexité énonciative de l'écriture collaborative, fréquente dans les pratiques scénaristiques du «cinéma d'équipe», à l'œuvre justement dans le travail des scénaristes d'En cas de malheur, Jean Aurenche et Pierre Bost, travail dans lequel s'immisce précocement le réalisateur luimême, Claude Autant-Lara.

Par cette approche multidimensionnelle qui emprunte à la génétique sa terminologie et sa méthode, l'essai s'écarte autant d'une conception simpliste de l'adaptation comme translation d'un médium vers un autre, que d'une vision seulement formaliste, qui réduirait l'encodage filmique à une traduction sémiotique, ligne à ligne, du langage littéraire du roman. L'adaptation suppose l'altérité : «La recherche "d'équivalences" vilipendée par Truffaut se situe bien au principe de la méthode des adaptateurs, mais on peut dire qu'elle est en fait requise dans toute démarche de transposition ou de traduction visant à conserver le texte premier comme référent principal tout en l'adaptant à un langage, à des intentions de représentations et un public-cible autres » (p. 322). De façon à la fois ambitieuse et instrumentée sur le plan critique, il s'agit d'ouvrir le «chantier scénaristique» du film, sorti le 17 septembre 1958, en soulignant les virtualités, les tâtonnements et les repentirs au cours de la préparation d'un canevas protéiforme qui débute le 8 avril 1957. Le spectacle de la genèse en acte est d'autant plus fascinant qu'il tient compte des voix multiples, convergentes, complémentaires ou divergentes de l'équipe du film, du premier scénario jusqu' au tournage, en studio et dans Paris, du 4 novembre 1957 au 4 février 1958. L'entreprise de dépliement des textes et des voix de la genèse de ce film en particulier échappe aussi à la logique simplificatrice et généraliste du « manuel»,

2. Nicole Marchand-Zañartu et Marie-Haude Caraës, Images de pensée, op. cit. (*) Université Sorbonne Nouvelle-Paris 3. 